\title{
Wavelet Packet Decomposition Based Traversable Terrain Classification for Autonomous Robot Navigation
}

\author{
K. K. SoundraPandian and Priyanka Mathur
}

\begin{abstract}
Autonomous robot must possess the capability to traverse intelligently on various terrains that may largely affect the performance and controllability of the vehicle. To enable autonomous navigation on natural unknown terrain, the traversal difficulty must be evaluated initially. This is accomplished by real-time assessment and classification of terrain for traversability analysis. A technique for terrain classification for traversability assessment of terrain by extracting the textural features from visual sensing of terrain data using wavelet packet decomposition (WPD) is presented in this paper. Clustering is used to classify the terrain describing its suitability for navigation. And further we have presented a fast and optimum algorithm for path planning of robot on the assessed navigable terrain.
\end{abstract}

Index Terms-Terrain classification, real-time traversability assessment, autonomous navigation, wavelet packet decomposition, clustering

\section{INTRODUCTION}

Autonomous robot navigation with the capability to perceive the surrounding environment of the robot enhances the efficiency and safety of the robot. An important functionality of an autonomous robotic system is real-time assessment and quantification of terrain for safe navigation along a path to a designated goal point. For safe autonomous navigation while traversing, a robot must possess onboard intelligence and the capability to perceive the terrain ahead so that it can avoid hazardous areas by discriminating the negotiable regions for traversal thereby optimizing its speed. This is accomplished by identifying the underline terrain that directly contributes to traversal difficulty. Enabling a mobile autonomous robot to determine in real time what kind of terrain it is facing has some great benefits in regard to the efficiency and safety of the robot. Terrain classification is fundamentally employed in Department of Defense for military surveillance, target tracking applications and in particular in NASA and ISRO for robotic planetary space explorations in the areas such as object recognition, detection of changes in terrain, local obstacle avoidance navigation and path planning by traversability assessment. A robot able to classify the terrain

Manuscript received September 9, 2012; revised October 15, 2012.

K. K. Soundra Pandian is with the PDPM-Indian Institute of Information Technology, Design and Manufacturing Jabalpur (e-mail: soundra.pandiankk@gmail.com).

Priyanka Mathru is with the Ansal Institute of Technology, Gurgaon, India (e-mail: priyanka10@gmail.com). ahead can optimize its speed for the terrain (drive slower on rough terrain and faster on smooth terrain) or avoid potentially hazardous areas, such as stretches of sand in which a ground-based robot could become stuck. Lack of terrain knowledge suspends reliable navigation and traversal to the goal successfully as exemplified by the NASA's Mars exploration rover in 2006 which became entrenched in loose drift material and remained stationary for several weeks [3]. Terrain classification to detect derivable ground for robot provides adaptability to control by optimizing its speed and planning strategies to avoid hazardous areas thereby improving its efficiency and safety.

The rest of the paper is organized as follows. A review of the related research work in the area of terrain classification and path planning using traversability analysis of terrain is given in section 2. Section 3 elucidates system overview and our methodology. In Section 4, path planning based on classified terrain is described. Section 5 presents our results and finally section 6 deals with conclusion and our future work.

\section{PROLOGUE ASSESSMENT}

Terrain classification methods provide semantic descriptions of the physical nature of a given terrain region. Terrain traversability based on terrain classification and path planning of terrain-adaptive robots have been addressed by a number of researchers. Howard et al [1] introduced the Fuzzy Traversability Index Algorithm, which used visual intensity levels to determine the terrain characteristics such as the roughness, slope, and discontinuity. Olson et.al. [2] proposed a method based on visual terrain mapping for Mars rovers. Using a visual stereo imaging fusion technique, they have demonstrated a reliable method for high fidelity terrain mapping and robot world perception modeling. Iagnemma et al. [3] classified terrain based on analysis of vibrations arising from robot wheel -terrain interaction. Vandapel et al. [4] categorized ladar data points as either clutter, linear or surface using range feature. In Manduchi et.al. [5] used a combination of color camera images and ladar data to detect and classify obstacles, with the detection done via ladar and classification using camera. Shirkhodaie et.al. [6] used visual terrain modeling using soft classifiers like rule based and neural networks. Machine learning methods were employed by Wolf et. al. [7] using 2 D laser range finders. Range information generates point clouds which are classified into navigable and not navigable area using hidden markov models (HMM).

Another line of research for terrain classification is to use 
the interaction between the terrain and the locomotion system for classification purposes as classified terrain based on analysis of vibrations arising from robot wheel -terrain interaction Kelly et al. [8] utilized multispectral imaging, different color spaces and their distribution statistics is used by Dima et al. [9] because many major terrain types possess distinct color signatures. A.Birk et al. [10] processed the range data obtained from Laser Range Finder by a Hough transform with three dimensional parameter spaces for representing planes and classified the terrain by Decision tree. Pandian et.al. [11] used textural gray level cooccurrence features with crisp rule based classifier to classify the terrain into several regions of navigable and not navigable area.

\section{Methodology}

\section{A. System Overview}

Terrain classification method extracts the relevant features which should be easily computed, robust, insensitive to various distortions and variations in the sensor data. The proposed terrain classification method consists of initially dividing the terrain image into finite number of sub frames where each frame represents a small portion of the actual terrain called the sub terrainian region. Texture features are then extracted from each sub image which is fed to the classifier that classifies the given sub frame into either navigable or not navigable region as shown in figure 1 . Texture is a measure of the local spatial variation in image intensity. The attributes of texture include contrast, variance, energy, and entropy etc. We compute features using wavelet packet decomposition and feature vector thus obtained are fed to the terrain classifier which uses $\mathrm{k}$ means clustering technique. After the classification terrain assessment is done for planning the navigation strategy of autonomous robot.
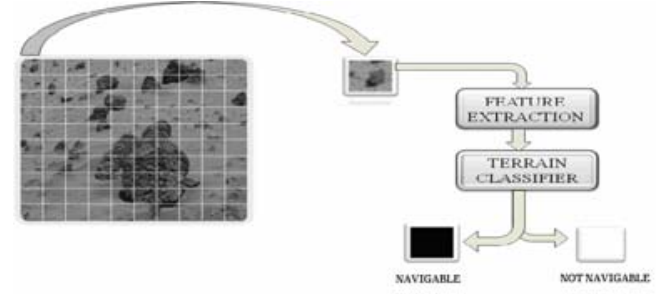

Fig. 1. Design flow of terrain classification

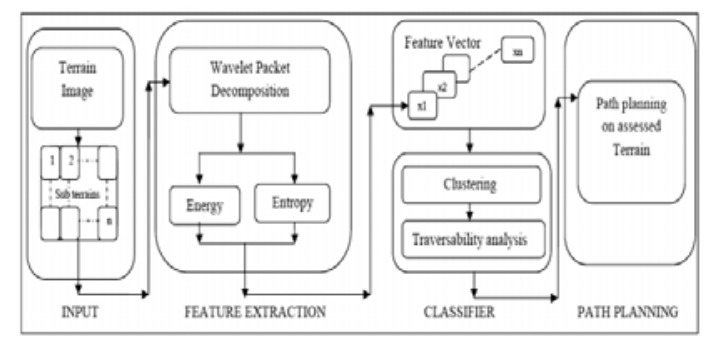

Fig. 2. Architecture of terrain classification system

\section{B. Feature Extraction}

Our approach to obtain feature vector is to apply wavelet packet decomposition on each sub terrain image. Feature database is created by using wavelet statistical features which contains energy values of leaf nodes of the wavelet packet decomposed image at level 3. Wavelet packet decomposition is an extension of the discrete wavelet transform Wavelet packet decomposition (WPD) (sometimes known as just wavelet packets) is a wavelet transform where the signal is passed through more filters than the Discrete Wavelet Transform (DWT). In the WPD, both the detail and approximation coefficients are decomposed using high pass $(\mathrm{h}[\mathrm{n}])$ and low pass filter $(\mathrm{g}[\mathrm{n}])$ respectively as shown in fig. 3 Instead of just decomposing the low frequency component, it is therefore possible to subdivide the whole time-frequency plane into different time-frequency pieces.

In order to minimize the computational requirement we have used mean, standard deviation of energy and entropy of the coefficients at the terminal nodes to achieve a separation of features for different classes. In addition, we have used another feature set using gray level co-occurrence matrix [17] for comparative analysis. Texture measures can be computed from the gray level co-occurrence matrix such as contrast, entropy, correlation and energy.

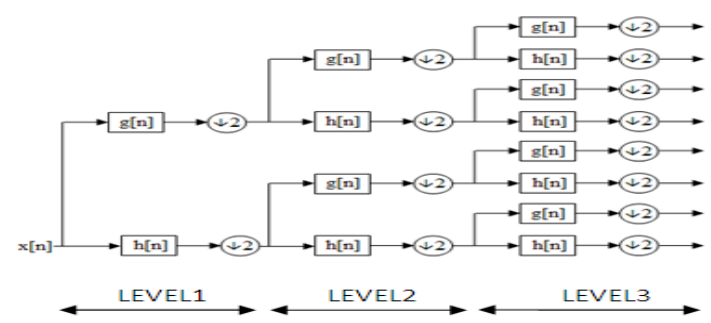

Fig. 3. Wavelet packet decomposition tree

An image is a matrix of pixel intensities, I $(i, j)$ We can define co-occurrence of image matrix as $P_{d}(i, j)$ such as every entry in co-occurrence matrix, $P_{d}(i, j)$ is difference in intensity between a pair of image pixels( $i$ and $j)$, that are distance d pixels apart in original image in a given direction. Energy associated with an image that is a measure of textural uniformity of an image is defined by equation

$$
\text { Energy }=\underset{i}{\sum} \sum_{j} P_{d}^{2}(i, j)
$$

Image Entropy is a measure of disorder of an image Entropy is inversely proportional to Energy and is defined by equation (1.2)

$$
\text { Entropy }=-\underset{i \text { j }}{-\sum \sum P_{d}(i, j) \log P_{d}(i, j)}
$$

The image texture contrast measures the amount of local pixels intensity variation within an image and is given by equation (1.3)

$$
\text { Contrast }=\underset{i j}{\sum \sum(i-j)^{2} P_{d}(i, j)}
$$

We compute these gray level co- occurrence features from the terrain image which forms another feature set 2 for terrain classification. A comparative analysis has been between this feature set and feature set 1 extracted by obtained by wavelet packet decomposition.

\section{Classifier}

Clustering is the process of finding groups of objects such that the objects in a group will be similar (or related) to one another and different from (or unrelated to) the objects 
in other groups. In statistics and machine learning, the kmeans algorithm is clustering algorithm to partition $n$ objects into $\mathrm{k}$ clusters, where $\mathrm{k}<\mathrm{n}$. This technique is based on randomly choosing $\mathrm{k}$ initial cluster centers, or means. These initial cluster centers are updated in such a way that after a number of cycles they represent the clusters in the data as much as possible.

The k-means algorithm starts with $\mathrm{k}$ - cluster centers or centriodes. Cluster centriodes can be initialized to random values or can be derived from a priori information. Each data point then assigned to the closest cluster (i.e. closest centriodes). Finally, the centriodes are recalculated according to the associated data until convergence is achieved. The k-means clustering groups data vectors into a predefined number of clusters, based on Euclidean distance as similarity measure. The centriodes vector is the mean of the data vectors that belong to the corresponding cluster. In this paper we assume $\mathrm{k}$ equal to 2 as we have two classes of terrain navigable and not navigable for traversability assessment.

\section{Path Planning on Assessed Traversable Terrain}

Path planning algorithm developed for the classified terrain obtains optimally shortest path for the robot. A four connected flood fill algorithm is developed in order to start at the goal and assign the lowest value- zero (0) to that cell of the grid. The not navigable regions are assigned the highest values-infinity $(\infty)$. All the four connected cells, starting from the goal, are filled with the values just one more than its smallest neighbor till the obstacle is met or end of the grid is reached. After filling the grid the path is planned starting from source cell and following the values downhill to the goal as shown in figure 4(a) as proposed by the author [17].

\begin{tabular}{|c|c|c|c|c||c|c|c|c|c|}
\hline 2 & 1 & 0 & 1 & 2 & 2 & 1 & 0 & 1 & 2 \\
\hline 3 & $\infty$ & 1 & $\infty$ & 3 \\
\hline 4 & 3 & 2 & 3 & $\infty$ \\
\hline 5 & 4 & $\infty$ & 4 & $\infty$ & $\infty$ & 1 & $\infty$ & 3 \\
\hline 6 & 5 & 6 & 5 & 6 \\
\hline 4 & 3 & 2 & 3 & $\infty$ \\
\hline 5 & 4 & $\infty$ & 4 & $\infty$ \\
\hline 6 & 5 & 6 & 5 & 6 \\
\hline
\end{tabular}

(a)

(b)

Fig. 4. Grid based path planning from source (yellow) to goal (red) Path planning (a) without diagonal movement (b) with diagonal movement

Moreover it optimizes the path by incorporating diagonal movement also. For example the path is 5(source)-3-2-10 (goal) as shown in figure $4(\mathrm{~b})$. The algorithm determines the most suitable way point towards the goal in the navigable region that minimizes the number of traveling cells thereby giving the shortest path

\section{RESULTS}

Our terrain traversability assessment method was tested on two different image databases. One data set was compiled from NASA's mars imagery [15] and another is from IIITDM campus. .The images were of the size $320 \mathrm{x}$ 320 . We chose a sub window frames of size $32 \times 32$ for terrain sampling. For Mars surface scenes, primary terrain types that are believed to possess distinct traversability characteristics are: rocky terrain, composed of outcrop or large rocks; sandy terrain, composed of loose drift material and smooth mixed terrain .Examples of these terrains are shown in Fig. 5, Iagnemma et al. [3]

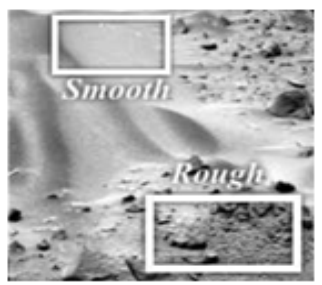

(a)

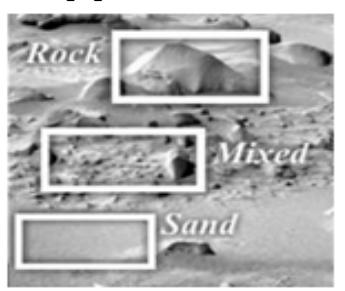

(b)
Fig. 5. Class distinction of Mars terrain (a) smooth and rough class (b) sand, mixed and sand class

Fig. 6 shows the result analysis of the martial terrain (Terrain image 1). As shown in figure 6(b) terrain image is divided into sub terrain frames. Classified terrain using features vector obtained from wavelet decomposition decomposition is shown in figure 6(c) where white color corresponds to not navigable region and black indicates the traversable region. In figure $6(\mathrm{~d})$ path planning is shown as developed by the MATLAB code where yellow cell indicates the source and red indicates the goal positions. The performance of a classifier can be measured by classification accuracies and speed. Accuracy is evaluated using Receiver Operating Characteristics (ROC) curve.

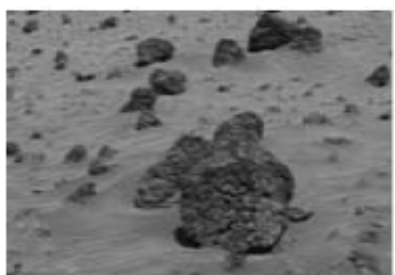

(a)

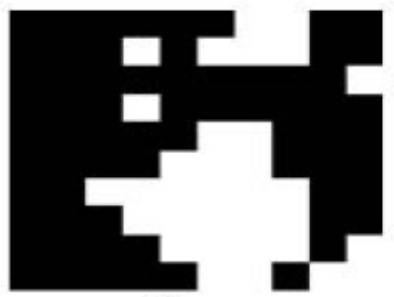

(c)

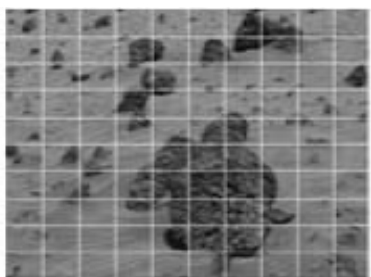

(b)

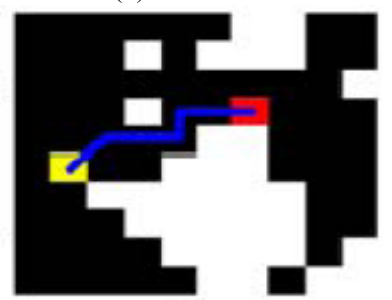

(d)
Fig. 6. (a) Terrain image $1 \quad$ (b) Sampled terrain (c) Classified terrain (d) Generated free path

Fig. 7 shows the result obtained on the real time terrain image (terrain image 12) obtained from IIITDM campus.

It summarizes how well the classifier has performed for that problem at different thresholds. It allows us to show graphically the trade off of each classifier between its true positive rate (the number of correct positive cases divided by the total number of positive cases) and its false positive rate (the number of incorrect positive cases divided by the total number of negative cases) by the total number of positive cases) and its false positive rate (the number of incorrect positive cases divided by the total number of negative cases) Figure 8(a) shows the ROC curves for terrain image 1.Here horizontal axis indicates the percentage of false positives and the vertical axis indicates the percentage of true positives. Blue curve line indicates the correct positive rate for not navigable regions and dotted green curve indicates correct classification rate for 
navigable regions. Higher the curve is towards the left higher is the correct classification rate as seen from the figure. Number of true positive case is $93.85 \%$ and false positive rate is $17.24 \%$. Figure $8(\mathrm{~b})$ is the ROC for real time terrain image 12. which shows that true positive rate is $91.67 \%$ for navigable region and 85.715 for not navigable region.

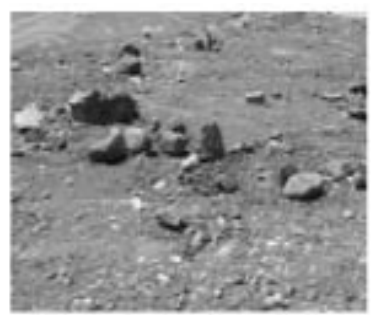

(a)

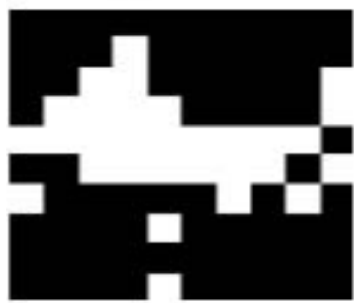

(c)

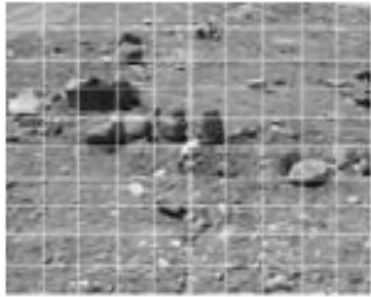

(b)

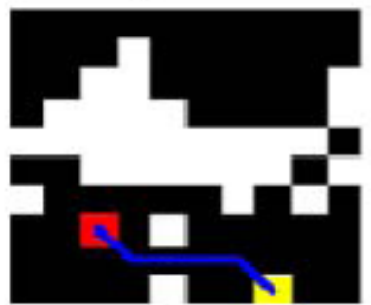

(d)
Fig. 7. (a) Terrain image 12 (b) Sampled terrain (c) Classified terrain (d) Generated free path

Fig. 9 (a) and 9(b) shows the ROC curves for terrain image 1 and 12 using gray level co-occurrence matrix as the feature vectors. Number of true positive case is $68 \%$ for terrain image 1 for navigable region and $90 \%$ for not navigable region with error rate of $30 \%$

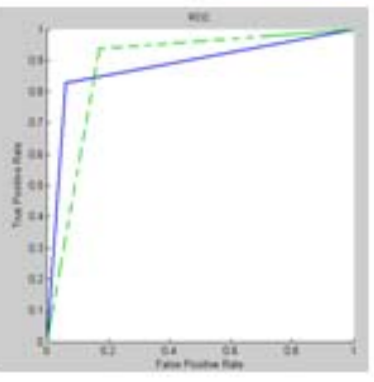

(a)

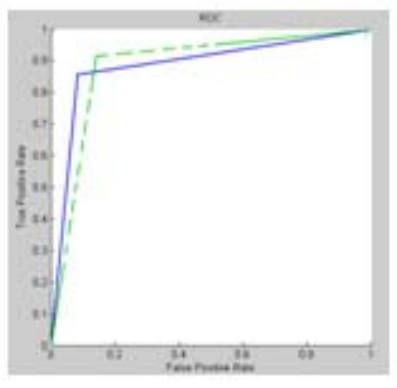

(b)
Fig. 8. (a) ROC curve of terrain image 1

(b) ROC curve of terrain image 12

For real time terrain image 12 , correct rate is $88.12 \%$ for navigable regions and error rate of $20 \%$ for not navigable regions. These results shows that feature set 1 using wavelet packet decomposition yields better results and higher success rate.

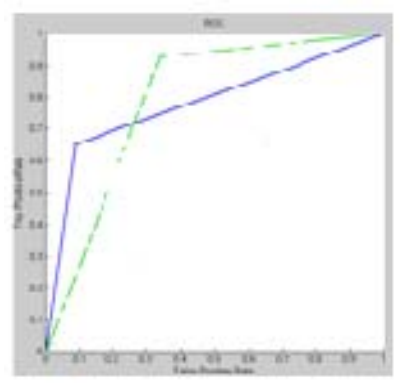

(a)

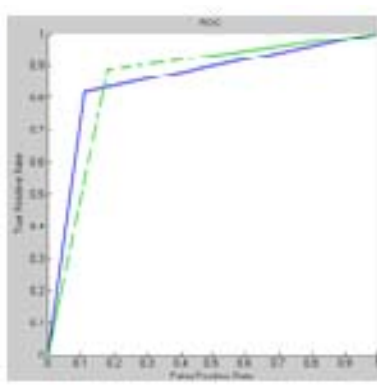

(b)
Fig. 9. ROC curve of (a) terrain image 1 (b) Terrain image 12

Table I. shows the classification accuracies of the classifier for $1100 \mathrm{sub}$ terrain regions of 11 martial terrain image NASA's mars exploration rover [15]. WPD is feature set obtained by wavelet packet decomposition and GLCM is the feature set obtained by gray level co- occurrence matrix. Table II. Represents correct classification rate for 900 image regions of 9 terrain images obtained from real time IIITDM campus.

Each image has hundred sub terrain regions which are classified as either navigable or not navigable. Figure 10 is the graph for correct classification rate for each of 20 images.

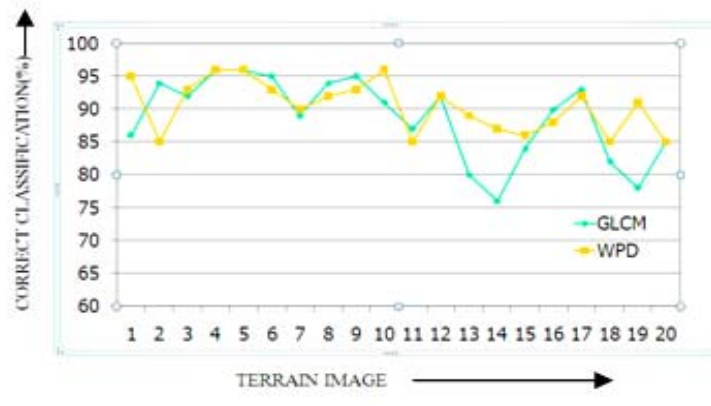

Fig. 10. Correct classification rate

TABLE I: CORRECT CLASSIFICATION FOR MAR'S TERRAIN IMAGES

\begin{tabular}{|c|c|c|}
\hline $\begin{array}{c}\text { Terrain Image } \\
\left(\begin{array}{c}100 \text { sub regions in } \\
\text { each image })\end{array}\right.\end{array}$ & WPD feature set & GLCM feature set \\
\hline 1 & 96 & 86 \\
\hline 2 & 95 & 94 \\
\hline 3 & 93 & 92 \\
\hline 4 & 96 & 96 \\
\hline 5 & 96 & 96 \\
\hline 6 & 93 & 95 \\
\hline 7 & 90 & 89 \\
\hline 8 & 92 & 94 \\
\hline 9 & 93 & 91 \\
\hline 10 & 96 & 87 \\
\hline 11 & 85 & 919 \\
\hline $\begin{array}{l}\text { Number of image } \\
\text { regions correctly } \\
\text { classified (1100 } \\
\text { subterranean regions) }\end{array}$ & 1014 & \\
\hline
\end{tabular}

TABLE II: CORRECT ClASSIFICATION (\%) FOR REAL TIME TERRAIN IMAGES OF IIITDM CAMPUS

\begin{tabular}{|c|c|c|}
\hline $\begin{array}{c}\text { Terrain Image } \\
\text { (100 sub regions in } \\
\text { each image) }\end{array}$ & WPD feature set & GLCM feature set \\
\hline 11 & 92 & 92 \\
\hline 12 & 87 & 76 \\
\hline 13 & 86 & 84 \\
\hline 14 & 88 & 90 \\
\hline 15 & 92 & 93 \\
\hline 16 & 85 & 82 \\
\hline 17 & 91 & 78 \\
\hline 18 & 85 & 85 \\
\hline 19 & 795 & 760 \\
\hline $\begin{array}{l}\text { Number of image } \\
\text { regions correctly } \\
\text { classified (900 } \\
\text { subterranean regions) }\end{array}$ & & \\
\hline
\end{tabular}




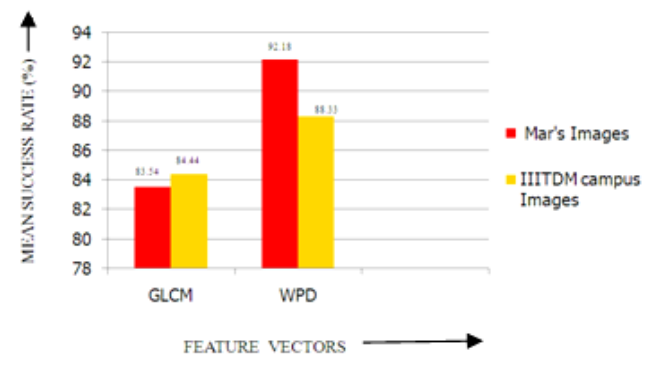

Fig. 11. Mean success rate

Results of classifier on 20 terrain images is shown having a total of 2000 sub terrain regions which are classified for traversability analysis. Mean success rate of the classifier using two feature sets is shown in figure 11.It is a graph for mean success rate $(\%)$ of terrain classification using different feature sets on two terrain image database.

\section{CONCLUSION AND FUtURE ASPECTS}

In this paper we have presented terrain classification technique to identify the traversable regions for the unknown natural terrain for autonomous robot navigation. Proposed approach classifies the unknown terrain describing its suitability for navigation by extracting the textural features from visual imagery of terrain data using wavelet packet decomposition. Comparative analysis has been done using two different feature sets on different terrain images. The mean success rate of terrain classifier using wavelet decomposed feature vector is higher than feature of cooccurrence matrix. For path planning we have used a flood filling algorithm that is both fast and shortest in generating free path in classified sampled terrain. The Classifier and path planning is developed using MATLAB. In future, this line of inquiry can be continued to develop improved terrain classifier that will able to differentiate more than two classes (navigable or not navigable) and should identify sandy, rocky and muddy terrain in the not navigable region.

Also, addition feature could be used to improve the classification accuracy. Further, the effects of employing different wavelets and alternative classifier architecture (such as support vector machines) to yield better classification accuracies on the terrain classification will also be investigated.

\section{REFERENCES}

[1] A. Howard, E. Tunstel, and D. Edwards, "An Intelligent Terrain Based Navigation System for Planetary Rovers," in the Joint 9th IFSA World Congress and 20th NAFIPS Int. Conf, pp. 7-12, Vancouver, B.C,Canada, July 2001
[2] C. F. Olson, L. H. Matthies, J. R. Wright, R. Li, and K. Di, "Visual Terrain Mapping for Mars Exploration," IEEE Aerospace Conference, pp. 1176, 2003.

[3] K. Iagnemma and A Brooks, "Self-Supervised Classification for Planetary Rover Terrain Sensing," IEEE Aerospace Conference, March 2007.

[4] N. Vandapel, D. F. Huber, A. Kapuria, and M. Hebert, "Natural Terrain Classification using 3-D Ladar Data," in Proceedings of the 2003 IEEE International Conference on Robotics and Automation, pp. 5117-5122

[5] R. Manduchi, A. Castano, A. Talukder, and L. Matthies, "Obstacle Detection and Terrain Classification for Autonomous Off-Road Navigation," Autonomous Robots, vol. 18. pp. 81-102

[6] S. R. Amrani and N. Chawla, "Traversable Terrain Modeling and Performance Measurement of Mobile Robots," Performance Metrics for Intelligent Systems Workshop, NIST, August 24-26, 2004.

[7] D. F. Wolf, D. Fox and W. Burgard, "Autonomous Terrain Classification Using Hidden Markov Models," in Proceedings of the 2005 IEEE International Conference on Robotics and Automation.

[8] A .Kelly, et al. "Toward Reliable off Road Autonomous Vehicles Operating in Challenging Environments," The International Journal of Robotics Research, vol. 25, no. 5, 2006.

[9] C. S. Dima, N. Vandapel, and M. Hebert, "Classifier Fusion for outdoor obstacle detection," in Proceedings of the IEEE International Conference on Robotics and Automation (ICRA)1, 2004.

[10] A. Birk, T. Stoyanov, and Y. Nevatia, "Terrain Classification for Autonomous Robot Mobility :from Safety, Security Rescue Robotics to Planetory Explorations," in Proceedings of the IEEE International Conference on Robotics and Automation (ICRA), 2008

[11] K. K. S. Pandian and P. Mathur, "Traversability Assessment of Terrain for Autonomous Robot Navigation," in Proc. of International Multi-Conference of Engineers and Computer Scientists - IMECS, Hong Kong, vol. II, pp. 1286-1289, March 2010

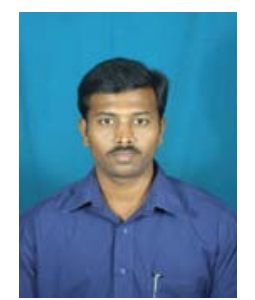

K. K. Soundra Pandian. This author became a Member (M) of IAENG in 2009, Member (M) of IACSIT in 2010. Born in chennai, tamilnadu. Graduate degree in electronics and communication engineering and post graduate degree in the applied electronics from university, chennai. Worked as Service Engineer from June 1998 to January 2001.Worked as Project Design Engineer from November 2001 to March 2003) in Centre for Electronics Design end Technology of India -Ministry of Communications and Information Technology). Worked as Research Engineer Grade II from July 2003 to December 2007 in Department of Mechanical Engineering, Indian Institute of Technology Kanpur, Uttar Pradesh. Working as Research Engineer Grade I from January 2008. Got Scientist ' $\mathrm{C}$ ' on deputation basis in the Ministry of Science and Technology, Department of Scientific \& Industrial Research in August 2010. K. K. Soundra Pandian (Mr.) is Member of International Association of Computer Science and Information Technology (IACSIT Membership No.: 80338135), Member of International Conference on Machine Learning and Computing (ICMLC) 2011: Peer Reviewer: Review Code: VIP-ICMLC-C01126, Member of International Conference on Mechanical and Electrical Technology (ICMET 2011): Reviewer /Committee, Committee Code: EN-C01126, International Conference of computer Technology and Development (ICCTD 2011): Technical committee, Committee Code: EN-D0357, International Conference on Signal Acquisition and Processing (ICSAP 2011Singapore, 26th -28th February 2011): Session Chair. The area of Image Processing Embedded systems - VLSI, ASIC \& FPGA design and Micro Electronics. 